\title{
Enabling Access to British Birth Cohort Studies: A Secure Web Interface for the NSHD (SWIFT)
}

\author{
Paul A. Watters, Diana Kuh, Susan Latham, Imran Shah \& Kevin Garwood \\ MRC Unit for Lifelong Health and Ageing \\ 33 Bedford Place \\ London WC1B 5JU \\ UK
}

\begin{abstract}
This paper provides an overview of the new Secure Web Interface for the Medical Research Council (MRC) National Survey of Health and Development (NSHD), which is a webbased data and metadata access system for the longest-running longitudinal study of health in the world [1]. Accessing NSHD metadata and data has often been a challenge for external (nonMRC) users, because the underlying data and metadata formats have changed dramatically in 63 years of operation, and because the processes involved in metadata search and data access were manual and usually required "on-site" access [2]. The design goals of the SWIFT include maintaining confidentiality and privacy of study members, enabling metadata search access to internal (MRC) and external users, facilitating data downloading and extraction from a range of underlying formats, and implementing procedures to ensure compliance with the governance policies of the MRC and the NSHD's governance panel. This paper details some of the challenges and successes of the MRC pathfinder "Data Access Project" to enable access using SWIFT while protecting the study members and their data.
\end{abstract}

Keywords-public health, secondary data access, governance, security policy

\section{INTRODUCTION}

The Medical Research Council (MRC) National Survey of Health and Development (NSHD) is an ongoing cohort study of a representative sample of 5362 men and women born in England, Scotland and Wales in a week in March 1946 and followed up 21 times since birth. The study's scientific strengths are the range and depth of prospective data on a) physical growth and development, and changes in adult body size and physical function, b) cognitive development and cognitive ageing and c) lifetime physical and mental health and health-related behaviours and d) indicators of the physical and social environment [3]. Buccal and blood samples were collected at age 53 years and blood and buccal DNA for 2,900 study members is held in the NSHD DNA repository [4].

The MRC Data Preservation and Sharing Initiative [5] aims to make anonymised scientific data more widely available across the research community. MRC identified NSHD as a priority pathfinder project on grounds of richness and quantity of data and is funding a NSHD Data Access Project. The objective is to develop a new NSHD data repository that integrates varied sources of data, metadata and semantic classifications in a web-based system to facilitate data discovery and use. A new set of governance arrangements for sharing data with external scientists has been developed as part of the NSHD Data Access Project. The new system will manage the data access application and review processes and integrate them with the discovery and secure downloading of data. This web-based system should be operational in 2010 and will facilitate and manage the expected growth in the use of NSHD data by bona fide researchers with high quality scientific research projects, whilst protecting data security, confidentiality and the reputation of the study.

For new, cross-sectional studies which have a relatively short data collection period, and which can be designed with new data management and metadata access principles and platforms in mind, providing external access should be relatively straightforward. Such a system might be built using an XML schema, mapped onto relational database tables, and easily integrated into a web-based system using a consistent set of descriptors, and deployed using a modern application server and operating system platform. However, since the NSHD has been actively collecting health information for 63 years, the underlying data formats, storage systems and media, operating systems, and searching capabilities are firmly rooted in the history of modern computing, i.e., also in the post-war period. The difference for the LHA - compared to finalised studies - is that data collected and stored in 1946 are still being actively utilised for scientific purposes, unlike many other systems created in 1946 or since. Thus, while the NSHD is a most significant data set in its own right, it also provides an instructive case study in data management issues, especially as secondary data access becomes entwined with the modern distributed computing fabric, comprising elements like "the cloud", "the grid", "the semantic web", "web 2.0", and so on (see [6] for a review).

\section{WEB-BASED DATA ACCESS}

The design goals of SWIFT are to provide:

- metadata search access to internal and external users, using both unstructured and structured search functions

- a mechanism to implement governance procedures around data access 
- a means of gathering together groups of variables in "shopping baskets", using an e-commerce metaphor

- a means of downloading data files with relevant metadata

- $\quad$ the necessary safeguards to protect the confidentiality and privacy of study members.

\section{A. Users and Roles}

The following access levels have been defined in the system:

- Level 1 access is publicly granted to members of the public. A general description of the study's holdings is available to Level 1 users.

- Level 2 access is intended for bona fide researchers. Access must be applied for, and - if granted - will provide unrestricted access to study metadata.

- Level 3 access provides unrestricted access to a preapproved set of variables detailed in a proposal, which has been approved through the NSHD's governance processes.

- Level 4 access will provide unrestricted access to study data for internal (i.e., MRC staff) users.

As part of the governance policy, SWIFT users (at Level 2 and above) can be asked to review access proposals. Reviewer access can be granted to users by the LHA Director. Both internal and external users can be reviewers.

There are four possible user types defined within the system:

- $\quad \operatorname{admin}$ - user is able to approve Level 2 user requests and Level 3 project proposals

- internal - user is granted unrestricted access to variables (intended for LHA team members only)

- $\quad$ external - user must apply for Level 2 and Level 3 access by completing the registration form and research proposal respectively.

- manager - the repository manager who is responsible for data and system management.

\section{B. User Functional Description}

The basic functions provided by SWIFT for external users are summarised briefly below:

- $\quad$ Registering as a new user (internal or external)

- Authenticating using the credentials supplied during the registration phase

- $\quad$ Searching the study metadata, including by keyword, category and year of collection

- Adding/deleting variables to a "shopping basket" of variables
- Creating a research proposal for (Level 3) data access using variables stored in the shopping basket

- Checking the status of Level 3 access requests

- Downloading data files once Level 3 access has been approved

- Securely logging out of the system

The menu system also provides for the following advanced functions:

- $\quad$ Managing documents uploaded as part of the Level 3 application process, including ethics approvals and/or relevant research papers

- Reviewing previous shopping baskets (if available)

- Reviewing baskets shared by others, and adding selected variables to the current shopping basket

- If the user is an approved reviewer, they may also be asked by the Director to review proposals and make recommendations based on the MRC LHA governance process

- Updating contact details

- Changing passwords

The full functionality of these features is too complex to describe here. However, a simple review of searching by keyword is provided, as an example. The NSHD metadata can be searched by keyword, year of collection or category, or a combination of all three search types, including:

- Search by keyword (including variable name and description)

- Search by year of collection

- Search by category

- Search by keyword and year

- Search by keyword and category

- Search by year and category

- $\quad$ Search by keyword, year and category

To search by keyword, you enter a search term, which will be matched against a variable name and description. You can also perform a soundex search on the term by checking the relevant box. Note that there is a maximum of 60 characters in the search term box.

When the Search button is clicked, the results are displayed as shown below: 


\section{Level 2 Access - Search}

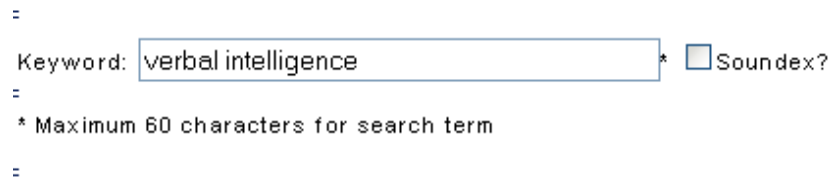

Figure 1. Variable search by keyword

The variable name, description and year of collection are displayed. The order of display is based on two factors: frequency on variable occurrence in all previous shopping baskets (from all users), and year of collection:

\begin{tabular}{|c|c|c|c|c|}
\hline \multicolumn{5}{|c|}{ Multiple Add } \\
\hline Variable & Description & Year & \begin{tabular}{|l}
$\begin{array}{l}\text { Add to } \\
\text { Basket? }\end{array}$ \\
\end{tabular} & $\begin{array}{l}\text { Multiple } \\
\text { Add? }\end{array}$ \\
\hline NV11R & education tests - Non Verbal Intelligence at 11 years - edited Raw score & 1957 & \pm & $\square$ \\
\hline $\mathrm{V11R}$ & education tests - Verbal intelligence at $11 \mathrm{yrs}$ - edited Raw score & 1957 & \pm & $\square$ \\
\hline$\underline{N \vee 15 R}$ & education tests - Non Verbal intelligence at 15 years (edited Raw score) & 1961 & \pm & $\square$ \\
\hline $\mathrm{Y} 15 \mathrm{R}$ & education tests - Verbal Intelligence at $15 \mathrm{yrs}$ - edited Raw score & 1961 & \pm & $\square$ \\
\hline NV11R57 & education tests Non-Verbal intelligence at 11 years raw score & 1957 & \pm & $\square$ \\
\hline$Y 11 R 57$ & education tests Verbal Intelligence at 11 yrs Raw score & 1957 & \pm & $\square$ \\
\hline NV15R61 & education tests Non-Verbal intelligence at $15 y$ r s raw score & 1961 & \pm & $\square$ \\
\hline NV1561 & education tests Non-Verbal intelligence at $15 \mathrm{yrs} T-$ score $x-?=50, S . D=10$ & 1961 & \pm & $\square$ \\
\hline Y15R61 & education tests Verbal intelligence at $15 \mathrm{yrs} R$ aw score & 1961 & \pm & $\square$ \\
\hline$\$ 1561$ & education tests Verbal intelligence at $15 \mathrm{yrs} t-$ score $x=50$ s.d $=10$ & 1961 & \pm & $\square$ \\
\hline NV1157 & education tests Non-Verbal intelligence at 11 yrs $T$ - score $x-?=50 s, d=10$ & 1957 & \pm & $\square$ \\
\hline$\$ 1157$ & education tests Verbal intelligence at $11 \mathrm{yrs} t-$ score $x=50$ s.d. $=10$ & 1957 & \pm & $\square$ \\
\hline NV11N & education tests - Non Verbal intelligence at 11 years - Normalised score & 1957 & \pm & $\square$ \\
\hline $\mathrm{V} 11 \mathrm{~N}$ & education tests - Verbal intelligence at 11 years - Normalised score & 1957 & \pm & $\square$ \\
\hline
\end{tabular}

Figure 2. Variable search by keyword results

Users are also given the option of adding a variable to the shopping basket by clicking the + symbol next to each variable, or adding a group of variables by checking the checkbox next to each desired variable, and then clicking the Multiple Add button.

To see variable metadata for each variable, users click on the hyperlinked variable name:

Level 2 Access - Variable Metadata

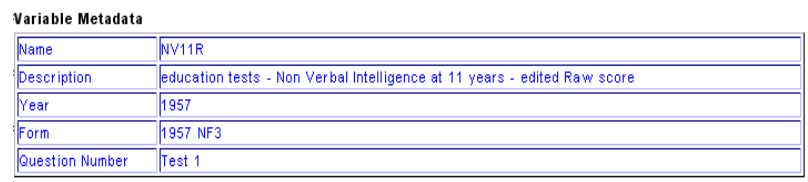

Add to basket

Value Labels

\begin{tabular}{|l|l|}
\hline Value & Label \\
\hline $0-40$ & Lowest to highest score [Non-verbal Intelligence test at 11 years - edited raw score] \\
\hline 99 & No questionnaire \\
\hline \hline
\end{tabular}

Figure 3. Variable metadata detail

Here, the name, description, year of collection, form number and question number are displayed, along with the physical reference to the variable in the data repository. In addition, most variables will also display the value labels for each variable.

Also, a set of the most related variables are displayed on the screen as well. Relatedness is measures by the frequency of cooccurrence of the target variables and all other variables from all previous shopping baskets (from all users). An example is shown below:

Related variables:
\begin{tabular}{|l|}
\hline Variable \\
\hline M11R \\
\hline V11R \\
\hline SEX \\
VOC11R \\
VOC8R \\
\hline SC8R \\
\hline
\end{tabular}

Figure 4. Related variable list

The full metadata record for each variable can be retrieved by clicking on the variable name, a shown below:

Repository Details
\begin{tabular}{|l|l|}
\hline Reference & D03 \\
\hline Column Start & 23 \\
\hline Column End & 24 \\
\hline $\begin{array}{l}\text { Code Book } \\
\text { Number }\end{array}$ & $62,(87)$ \\
\hline
\end{tabular}

Figure 5. Physical repository details

If the variable is a derived variable, then additional details of how the derivation was made will be displayed.

\section{Logical Architecture}

The essential features of the logical architecture are summarised below:

- External users interact with the SWIFT through a firewall and/or security device

- All connections between the web application and external users are made using the Secure Sockets Layer (SSL) for confidentiality

- Access to the data repository is also performed using SSL

- Access to the metadata repository is made using the Java Database Connectivity (JDBC) API

\section{Physical Architecture}

The essential features of the physical architecture are summarised below:

- The SWIFT application runs inside a Tomcat (Java) container, on a Windows server

- The metadata database (incorporating the data dictionary and derived data library) runs on a MySQL instance, on a Windows server

- The data repository runs on a Solaris server with SPSS 


\section{E. Platform}

SWIFT is a three-tier web application designed to minimise dependencies on new technological developments:

- The client tier consists of dynamically-generated HTML code, forms and JavaScript. Form validation is performed using JavaScript.

- The middle tier consists of a set of servlets and JSP pages that are executed by the Tomcat web container. A full Java EE application server is not required, and the application is not dependent on any third-party or evolving framework for persistence or presentation, such as EJB, Spring, Hibernate etc Standard SQL is used for all queries and table updates. Any Java application server or servlet runner that supports the servlet and JSP APIs can be used.

- The data tier consists of a set of tables which are stored in a MySQL database. No stored procedures are used. Any database that supports JDBC can be used there is no MySQL-specific code or configuration used.

Development has been performed using Macromedia Dreamweaver (client tier), NetBeans (middle tier) and MySQL admin tools (data tier).

\section{Metadata ENHANCEMENT}

In order to achieve the long-term strategy to enhance preservation of all NSHD data for sharing and re-use, the LHA has been planning and executing related activities, such as the standardisation of study metadata. The two key enhancements made have been the design and development of relational databases to store the study's data dictionary and details of derived variables.

\section{A. Data Dictionary}

Most information systems have a central data dictionary that contains key reference metadata for the data being held by an organisation. In the case of NSHD, this metadata has been recorded in a number of different ways over the course of the study. Examples of metadata held include:

- the study questionnaires

- an index to all of the questionnaire questions

- an index to the physical location of each variable

- a label for each variable

- a set of value labels for each variable

- one or more high-level categories for each variable

- sets of variables used in particular analyses or published in research papers, including derived data

Centralising and standardising all of this metadata has incurred a significant cost, however, making such structured data - and its content - available for SWIFT has made searching possible using terms (variable label, value label etc), year of collection, or category, or Boolean combinations of all three metadata types.

\section{B. Derived Data Library}

It is common to derive variables from others and, in prospective studies such as the NSHD, to examine their changes across the life course. An example is deriving the Body Mass Index (BMI), which is calculated from heights and weights. New technologies and metrics for measuring heights and weights may have introduced bias into the underlying variables. In addition, there are varied ways in which variables have been cleaned and transformed; thus documenting all factors relevant to the derivation is a non-trivial exercise. A more complex example is the age at menopause that is derived in NSHD from responses to a number of different questions given annually by women study members over a period of 8 years in midlife.

The derived data library supplements the data dictionary by providing full details of the derivation process, including:

- variable author

- date last updated

- title

- a summary of how the raw source variables are connected

- list of variables

- what information the derived variable provides and units of measurement, if appropriate

- the method used in derivation

- any raw variables/previously derived variables that were used in the derivation of the variable

- any problems encountered in derivation of the variable and how they were dealt with, e.g., setting a missing code to an implausible value, and noting why it was decided not a valid value

- descriptive statistics for the variable, and if the categorical, frequencies for the variable with the category labels

- reference details if the derived variable has been used in a published paper

\section{Categorisation}

Variables in the study may be associated with one or more high-level categories. The categorisation scheme is not a hierarchical ontology - indeed, public health is crying for the development of a standardised ontology [7] - however, the system has been used successfully for internal categorisation for a number of years. Since SWIFT has the ability to use any categorisation scheme, if integration with an external (public health or epidemiological) ontology was required, the integration could either be achieved through indirection (if the internal scheme was to be preserved) or a simple update of the underlying database records. Given the large number of 
variables, an automated categorisation scheme would be ideal, but would require an effective word-sense resolution mechanism to ensure that the correct "sense" of each word was identified in context [8].

The categories currently in use include:

- $\quad$ Response at each follow-up

- Anthropometry

- General health

- Wellbeing

- Respiratory health

- Cardiovascular health

- Musculoskeletal health \& physical capability

- Mental health

- Women's health

- Hospital admissions

- Medication

- Other health care

- Mortality

- Cognitive function

- Temperament/personality

- Nutrition

- Other health behaviours

- Puberty/fertility history

- Marital history

- Household structure

- Education

- Social class

- Employment history

- Other socioeconomic circumstances

- Social support/life events

- DNA samples

- $\quad$ Blood and urine samples

\section{GOVERNANCE}

New procedures established in 2007 have led to a faster turnaround on data requests than was previously possible. Proposals are reviewed by the LHA/NSHD Executive Team on a monthly basis and, if approved, external researchers are invited to create their own data file under the guidance of the NSHD Data Repository Manager. The role of SWIFT is to automate the current form-based approach with the goal of further reducing turnaround times. Now, the Director is able to make decisions about access - based on reviews by the
LHA/NSHD Executive Team or external experts - and all decisions can be logged and potentially audited.

Proposals are considered by the LHA/NSHD Executive Team (chaired by the Director and consisting of senior LHA scientists and NSHD operational heads) on a monthly basis. When projects are proposed that are similar to projects already approved or to LHA scientific programmes, researchers are encouraged to collaborate on a common project.

The proposal must meet the following criteria:

- No breach of confidence should be incurred, and the use should be within the bounds of consent given by cohort members, and within the MRC code of ethics.

- There should be no serious risk to the viability of the study through offence to cohort members from use of the data supplied.

- Proposal should be from a bona fide scientist with the necessary expertise to undertake the project. In the case of students applying for access, their supervisor must be a co-applicant and ensure that appropriate academic and data security guidance are provided.

- The science of the proposal should have been peerreviewed, and if not should be peer-reviewed by the Executive Team or their expert advisers. The aim is to attract high quality research proposals from external scientists. At a minimum, the science must be good enough not to bring the study into disrepute

SWIFT will manage the data access application and review processes and integrate them with the discovery and secure downloading of data. This web-based system should be operational in 2010 and will facilitate and manage the expected growth in the use of NSHD data by bona fide researchers with high quality scientific research projects, whilst protecting data security, confidentiality and the reputation of the study.

Current ethical approval covers data sharing with scientific collaborators on the basis of consents given previously by study members. Only rarely are NSHD data permitted to be sent outside the UK. In the future, it may be possible to offer SWIFT as a hosted service using a secure Terminal Services or similar. Combined with a hosted analytical package, this means that international researchers would be able to access data and perform analyses without the data having to leave the UK.

\section{DATA MANAGEMENT}

According to the governance policies and procedures detailed in Section IV, there are two situations where authorised metadata searching results in study data access:

- An internal (MRC staff member) user has a shopping basket of variables that they wish to download

- An external user has a Level 3 access request approved which has a list of authorised variables for download

In both cases, the data management workflow follows a similar path: 
- The metadata for each variable is gathered into a UNIX script which is generated on the application server. The generation of the UNIX script is non-trivial, since it involves generating SPSS import statements for several different underlying file formats, and it also requires the integration of many different items of metadata from the metadata database

- The UNIX script is automatically downloaded to the NSHD data repository

- Semantic script checking to ensure that access restrictions of variables are preserved, and that the variable list contained in the script matches the authorised variable list (which is uploaded to the NSHD data repository out-of-band).

- The UNIX script is executed against the data repository server, which in turn invokes SPSS to generate a portable SPSS output file, which contains fully labelled metadata for each of the variables authorized for access

- For external users, scrambling is performed on the study members' serial numbers, on a per-user basis, to preserve study member privacy by preventing collusion by users. Internal users do not use scrambled serial numbers since they are required to clean and maintain the original data records.

- $\quad$ The SPSS POR file is uploaded to SWIFT, and the user is notified that the file is available for download

This automated process takes around 10 seconds for a modestly-sized statistical analysis.

\section{SECURITY}

SWIFT implements strategies to achieve the security goals of confidentiality, integrity and availability.

\section{A. Confidentiality}

SWIFT only operates using the Secure Sockets Layer (SSL) which encrypts all web traffic between the user's web browser and the LHA web server. This means that the username, password, and any data that the user downloads from the web server can be intercepted by a third party, but not deciphered. Thus, the possibility that data will be "lost in the post" and accidentally disclosed is eliminated. Once data has been downloaded to a remote user's system, the LHA has developed a set of security guidelines to assist remote users in securing the data. Since each remote user is issued with a unique set of serial numbers for each case in the dataset, it is possible for the LHA to trace back any inappropriate disclosures to external users.

\section{B. Integrity}

SWIFT automatically checks that the variables requested during data download match those which were actually approved through the governance process, through an independent channel. These checks ensure that users are provided with the correct data, and also that data are not inappropriately disclosed to unauthorized users. The check also ensures that malicious users have not managed to subvert the protections present in the application tier, ie, scripts are checked for SQL injection attempts and all "reserved" SQL terms are blocked from data entry.

\section{Availability}

SWIFT runs on a highly-available server, with sufficient memory and redundant drives/processors to ensure continued operation in the event of a single component failure.

\section{DISCUSSION}

This paper provides an overview of SWIFT and the metadata search, data download and governance access policies that it implements. In addition, a review of non-functional requirements, such as security, has also been provided. The requirements and specification for the system have been developed using a modified version of the agile project management methodology, known as scrum, which involves presenting deliverables to end users and seeking their feedback on the prioritisation of new features. Development of the system is ongoing, and a number of enhancements - especially in the remote access area - are planned in the coming years.

The current implementation raises a number of technical and governance issues including whether external users should be able to download data to their own PCs for analysis, or whether an online analysis should be provided, where only analysis results can be downloaded. Providing an analysis service would prevent data proliferation and promote integrity, but would require an additional investment in server, network and software infrastructure. Conversely, if external users are allowed to download data, but their infrastructure is not as highly protected as the NSHD data repository, then the risk of inadvertent disclosure increases, particularly if external auditing of the security of their systems is not in place and may not be feasible.

To solve this problem, it would be helpful if a set of shared standards around data security in UK population health were adopted to enable the sharing of data to take place with as a few barriers as possible. One approach would be to adopt (or adapt?) the ISO 27001 standard for information security management.

The second barrier to widespread data access is the lack of a standard classification scheme and/or ontology for population health studies, which would make it easier to identify similar variables across different cohort studies, and/or provide the capacity to represent the different process involved in measuring, cleaning, and deriving data.

Future work must consider how to enable interoperability in a secure and semantically-sound way.

\section{REFERENCES}

[1] M. Wadsworth, D. Kuh, M. Richards, and R. Hardy, "Cohort profile: the 1946 national birth cohort (MRC National Survey of Health and Development)," International Journal of Epidemiology, vol. 35, pp. 4954, 2006. 
[2] W. Hilder, "60 Years of data curating a life course study, the Medical Research Council National Survey of Health and Development", http://www.dcc.ac.uk/docs/mdb-2005/hilder mdb 2005.ppt, 2005.

[3] D. Kuh, D., Y. Ben-Shlomo, J. Lynch, J. Hallqvist, and C. Power, "Glossary for life course epidemiology" Journal of Epidemiology \& Community Health, vol. 57, pp. 778-83, 2003.

[4] D. Kuh, "A life course perspective on telomere length and social inequalities in aging," Aging Cell, vol. 5(6), pp. 579-580, 2006.

[5] A. Sudlow, "Making better use of MRC-funded research data," Available

from www.mrc.ac.uk/Utilities/Documentrecord/index.htm?d=MRC003346
[6] M. Hardey, "Public health and Web 2.0," The Journal of the Royal Society for the Promotion of Health, vol. 128(4), pp181-189, 2008.

[7] L. Jorm, Su Gruszin, Tim Churches. A multidimensional classification of public health activity in Australia. Australia and New Zealand Health Policy 2009, 6:9.

[8] P. Watters, "Discriminating English Word Senses Using Cluster Analysis", Journal of Quantitative Linguistics, 9(1), 77-86 (2002). 